\title{
Operando Soft X-ray Spectroscopy Probing Chemical Transformation in Space and Time
}

\author{
Yi-De Chuang ${ }^{1}$, Per-Anders Glans ${ }^{1}$, Wanli Yang ${ }^{1}$ and Jinghua Guo ${ }^{1}$ \\ ${ }^{1}$ Advanced Light Source, Lawrence Berkeley National Laboratory, Berkeley, CA 94720, USA.
}

Advanced energy technology arises from the understanding in fundamental science, thus rest in large on in-situ/operando characterization tools for observing the physical and chemical interfacial processes. The energy materials and devices have been largely limited in a framework of thermodynamic and kinetic concepts or atomic and nanoscale. We have developed the in-situ/operando soft X-ray spectroscopy for the studies of catalytic and electrochemical reactions in recent years and overcome the challenge that soft $\mathrm{X}$-rays cannot easily peek into the liquid electrochemical cells under vacuum condition. Soft X-ray spectroscopic techniques offers unique characterization in many important energy materials of energy conversion, energy storage and catalysis in regards to the functionality, complexity of material architecture, chemistry and interactions among constituents within. We have demonstrated how to best use the in-situ/operando soft X-ray spectroscopy characterization techniques, including soft X-ray absorption spectroscopy (XAS) and resonant inelastic soft X-ray scattering (RIXS) to investigate the real electrochemical mechanism during the operation. The experimental results show that in-situ/operando soft X-ray spectroscopy techniques can further enhance the understanding of real reaction mechanism. provide element specific access to the local chemical states in liquids, gas-phase molecules, and at the liquid/solid and gas/solid interfaces [1-4] during the catalytic and/or electrochemical reactions.

An increasing number of experiments are performed in situ or in operando in order to study the fundamental mechanisms underlying complex processes such as electrochemical energy conversion under realistic conditions. The fundamental challenge, however, remains to bridge the spatial, temporal, and thermodynamic scales that are simultaneously relevant for the outcome of chemical reactions. A variety of techniques are capable of Angstrom spatial, attosecond temporal, or sub-meV energy resolution and, in the case of soft x-rays, elemental and chemical sensitivity. It is becoming apparent that the biggest challenges for experimentalists may now be the heterogeneity of chemical reactions in space and time. Often chemistry is based on statistical processes. Fluctuations in large ensembles lead to local conditions under which, at any given time, a small number of molecules can undergo chemical transformations. These conditions are only met for a very short period of time and only in a few specific locations. It is then and there where one needs the best possible spectral, temporal, and spatial resolution in order to capture the correlated intra- and inter-molecular dynamics that are at the heart of chemical transformations. The location and time, however, of statistical processes is, by definition, not known. The discrepancy between the spatiotemporal heterogeneity of chemical reactions and the tight spatiotemporal restrictions of virtually all probes for fundamental interactions is one of the biggest challenges for the study of "realworld chemistry."

We are developing the imaging RIXS to enable simultaneously probing the chemical transformations on multiple time and length scales [5]. An extension of this technique to facilitate the spatially and temporally resolved measurements of electronic dynamics will open a new direction for research in materials science and chemical transformation. The ability to simultaneously probe chemical transformation related to charge transfer and electron flow in "real-world" systems on multiple time (down to $1 \mathrm{~ns}$ ) and length (100 $\mathrm{nm}$ ) scales creates a unique facility, while key feature of the imaging RIXS is to simultaneously probe chemical transformations on multiple time and length scales [6]. 


\section{References:}

[1] A Braun et al, J. Phys. Chem. C 116 (2012), p. 16870.

[2] TS Arthur et al, Electrochem. Commun. 24, (2012), p. 43.

[3] JJ Velasco-Velez et al, Science 346 (2014), p. 831.

[4] LC Kao et al, Surface Science 702 (2020), p. 121720.

[5] Y-D Chuang et al, J. Synchrotron Rad. 27 (2020), p. 695.

[6] J Kern et al, Science 340 (2013), p. 491.

[7] This research used resources of the Advanced Light Source, a DOE Office of Science User facility under contract no. DE-AC02-05CH11231.
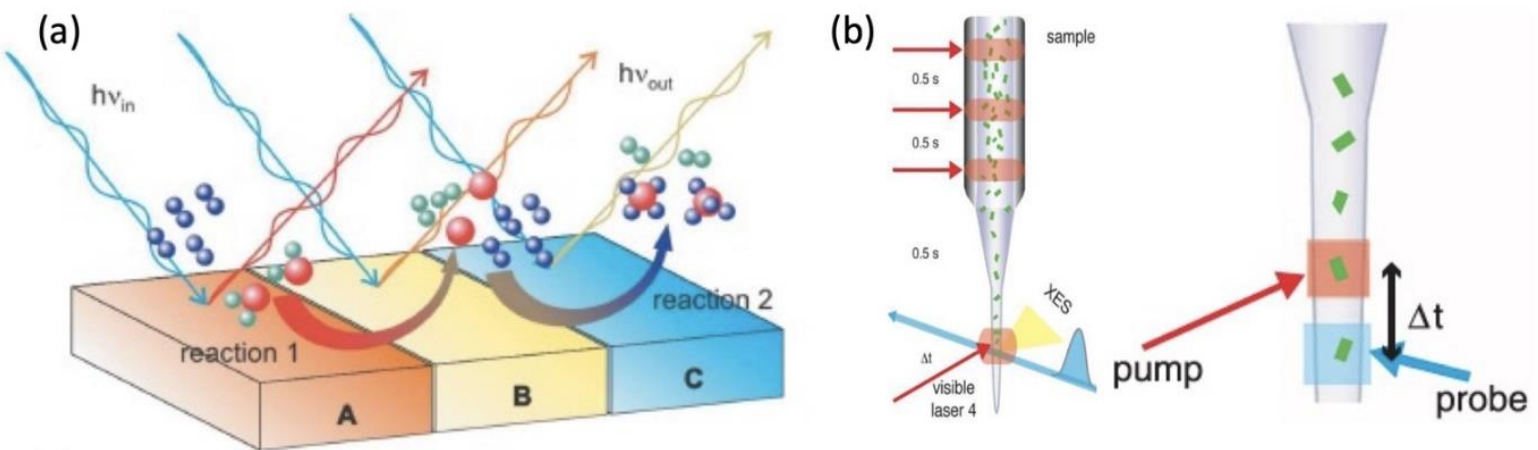

Figure 1. (a) Schematic illustration of a tandem catalyst consisting of three materials $A, B$, and $C$ that are tailored for reactions 1 and 2 [5]; (b) A proposed pump-probe scheme using micro-/nano-focused x-rays to elucidate not only the structure of the catalytic complex [6], but also the changes in the structure as it cycles through the intermediate states, leading to an understanding of the mechanism. 